ARTICLE

DOI: $10.1038 / s 41467-018-03614-0$

OPEN

\title{
A TAD boundary is preserved upon deletion of the CTCF-rich Firre locus
}

\author{
A. Rasim Barutcu (1) 1,2, Philipp G. Maass (1) 1, Jordan P. Lewandowski ${ }^{1,3}$, Catherine L. Weiner (D) ${ }^{1,2,3,4} \&$ \\ John L. Rinn (1) 1,2,3,4,5
}

The binding of the transcriptional regulator CTCF to the genome has been implicated in the formation of topologically associated domains (TADs). However, the general mechanisms of folding the genome into TADs are not fully understood. Here we test the effects of deleting a CTCF-rich locus on TAD boundary formation. Using genome-wide chromosome conformation capture $(\mathrm{Hi}-\mathrm{C})$, we focus on one TAD boundary on chromosome $\mathrm{X}$ harboring 15 CTCF binding sites and located at the long non-coding RNA (IncRNA) locus Firre. Specifically, this TAD boundary is invariant across evolution, tissues, and temporal dynamics of $\mathrm{X}$-chromosome inactivation. We demonstrate that neither the deletion of this locus nor the ectopic insertion of Firre cDNA or its ectopic expression are sufficient to alter TADs in a sex-specific or allele-specific manner. In contrast, Firre's deletion disrupts the chromatin super-loop formation of the inactive X-chromosome. Collectively, our findings suggest that apart from CTCF binding, additional mechanisms may play roles in establishing TAD boundary formation.

\footnotetext{
${ }^{1}$ Department of Stem Cell and Regenerative Biology, Harvard University, Cambridge, MA 02138, USA. ${ }^{2}$ Broad Institute of Massachusetts Institute of Technology and Harvard, Cambridge, MA 02142, USA. ${ }^{3}$ Department of Pathology, Beth Israel Deaconess Medical Center, Boston, MA 02215, USA.

${ }^{4}$ Department of Molecular and Cellular Biology, Harvard University, Cambridge, MA 02138, USA. ${ }^{5}$ Department of Biochemistry, University of Colorado, BioFrontiers Institute, Boulder, CO 80301, USA. Correspondence and requests for materials should be addressed to

A.R.B. (email: rasimbarutcu@fas.harvard.edu) or to J.L.R. (email: johnrinn@colorado.edu)
} 
T opologically associated domains (TADs) are units of chromosomes that are separated by regions known as TAD boundaries and exhibit higher frequency of physical contacts between genes and their cognate regulatory elements ${ }^{1,2}$. The organization of the genome into TADs is critical for coordinated transcriptional regulation, chromatin states, and DNA replication $^{2-4}$. The CTCF protein has been identified as one master organizer of this process ${ }^{5,6}$ and its orientation-dependent DNA binding has been implicated in establishing TAD boundaries ${ }^{7-15}$. However, several studies have yielded inconsistent results on the role of CTCF in TAD boundary formation. The depletion of CTCF affects cell survival and leads to global loss of TADs ${ }^{16}$. Although the deletion of a single CTCF site or of a minimal

a

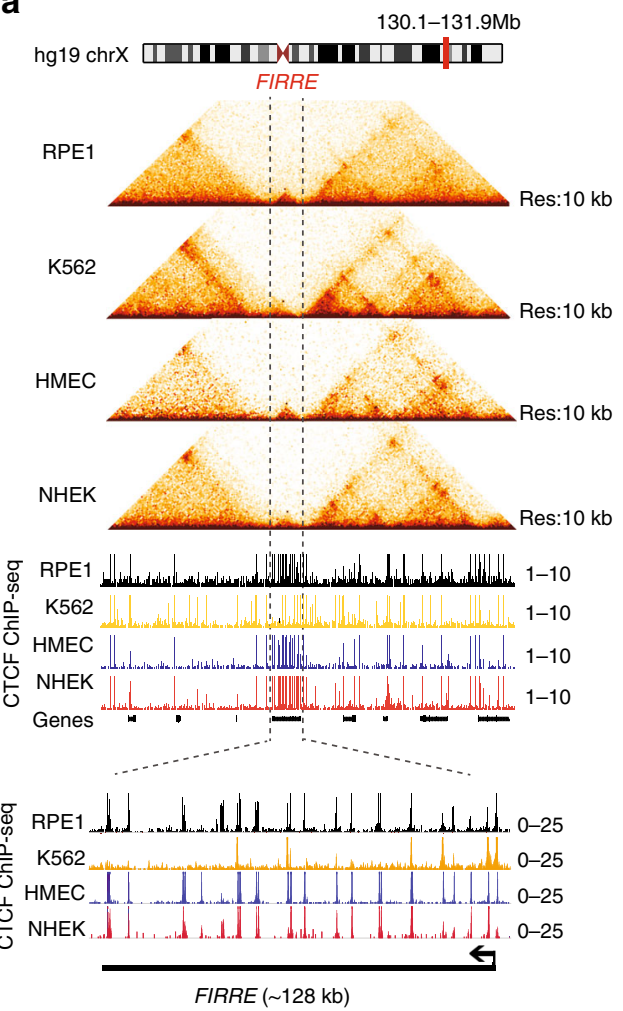

C

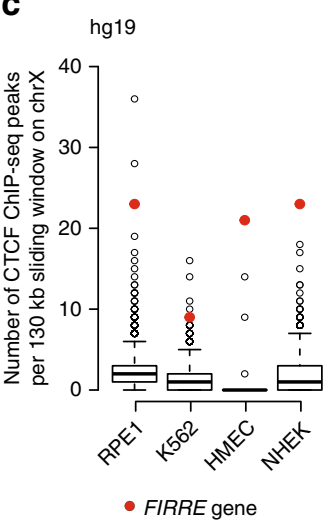

genomic region is sufficient to perturb a TAD boundary in some studies $9,17,18$; others reported that disruptions of a TAD boundary occur only upon deleting very large genomic regions (e.g., $200-400 \mathrm{~kb})^{1,18}$.

To address this conundrum, we focused on a locus on chromosome $\mathrm{X}$ that exhibits dense CTCF binding, and that harbors the Firre long non-coding RNA (lncRNA) ${ }^{19,20}$. The Firre locus topology is evolutionarily conserved across human and mouse, and displays enriched CTCF binding across many cell types ${ }^{19,20}$. More recently, Firre has been found to interact with the DXZ4 macrosatellite, a region located at the hinge of the mega-domain formation on the inactive $\mathrm{X}$ chromosome $(\mathrm{Xi})^{12,21,22}$. On $\mathrm{Xi}$, DXZ4 is at the anchor of a conserved super-loop formation
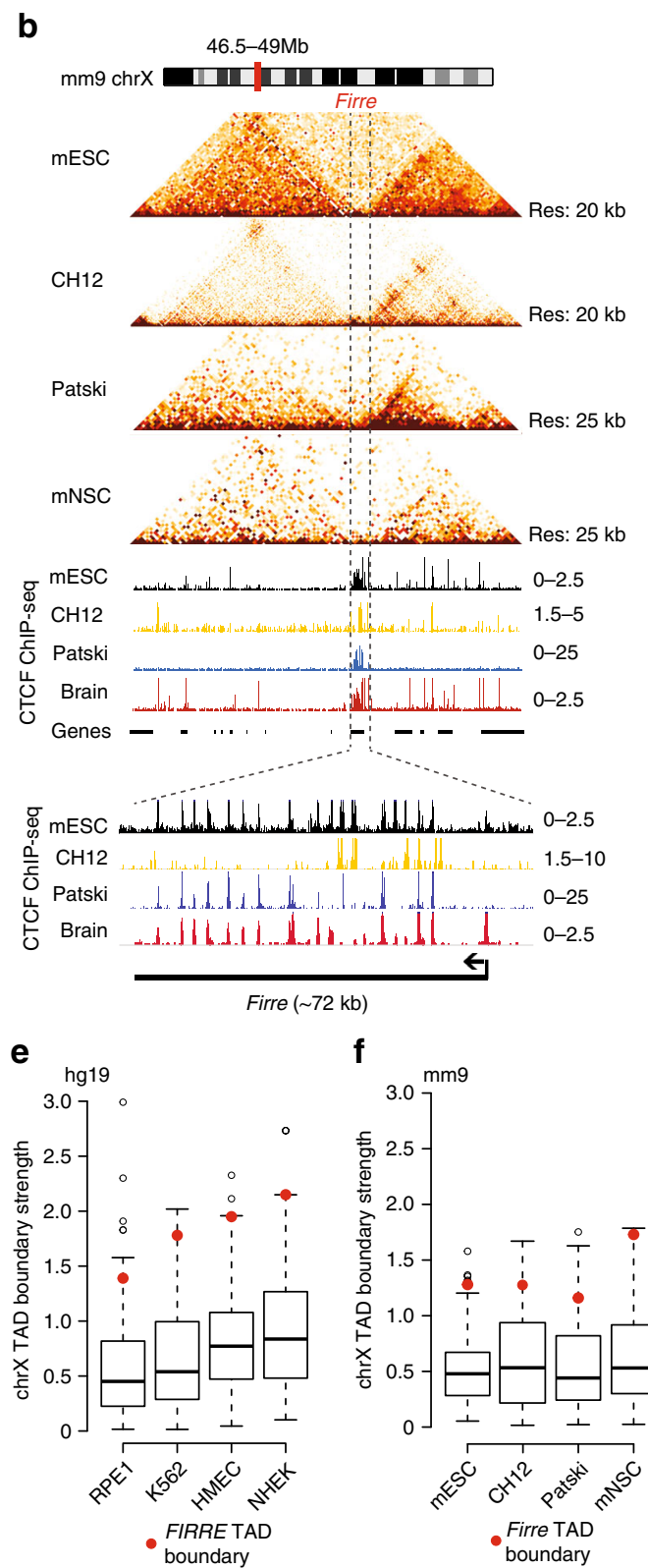

Fig. 1 Firre is consistently located at a TAD boundary and harbors several CTCF sites. a Human Hi-C heatmaps showing \pm 1 Mb of the FIRRE locus in RPE-1 (female), K562 (female), HMEC (male), and NHEK (unisex) cell lines (upper panel), and CTCF ChIP-seq signals across the Firre locus (lower panel). b Mouse $\mathrm{Hi}-\mathrm{C}$ heatmaps depicting the Firre locus in mESCs (male), $\mathrm{CH} 12$ (female), Patski (female), and mouse neuronal stem cells (mNSCs) (unknown sex, upper panel), and a zoom-in of the CTCF ChIP-seq signal (lower panel). c, d Box plot showing the number of CTCF peaks for each sliding window on the (c) human and (d) mouse chromosome $X$. The bin containing the human and mouse Firre genes is shown with a red dot. e, $\mathbf{f}$ Boxplot showing the TAD boundary scores for all the boundaries on the $X$ chromosome in different cell lines in (e) human and in (f) mouse. The TAD boundary that contains Firre is shown with a red dot. Error bars: s.d. 
a

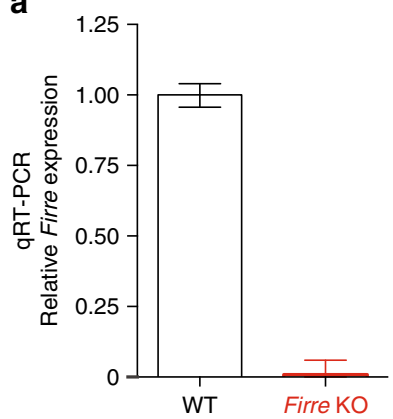

b

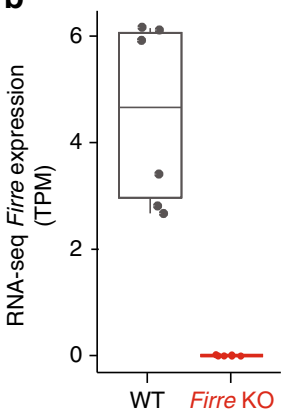

C

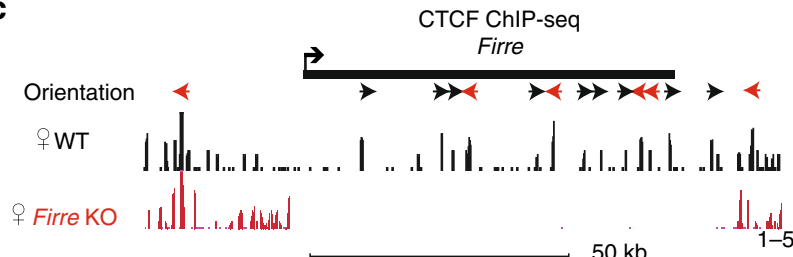

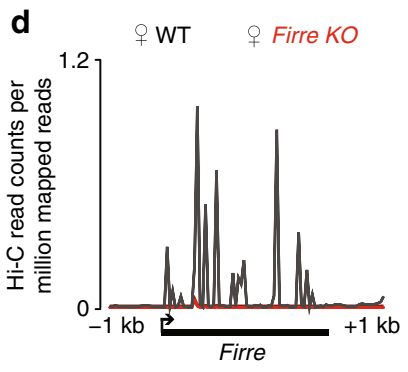

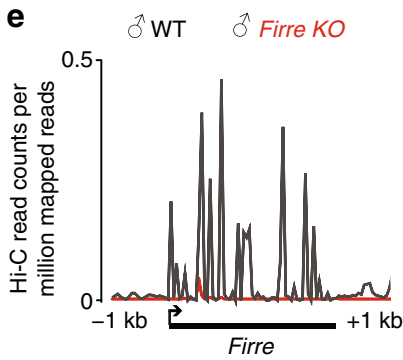

Fig. 2 Validation of Firre knockout in MEFs. a qRT-PCR analysis of Firre expression in wild-type and knockout MEFs. Error bars: s.e.m. b Plot showing transcripts per million (TPM) values for wild-type and Firre KO MEF RNA-seq. Error bars: s.d. c CTCF ChIP-seq signal tracks showing the complete loss of CTCF binding at the Firre locus in Firre KO MEFs (mm9, chrX:47.8-49 Mb). d, e Hi-C reads per million (RPM) values for the Firre locus in (e) wild-type and (f) Firre KO MEFs

involving the Firre locus, the inactive-X CTCF-binding contact element (ICCE), and a region termed "x75"23-25. Although abolishing the DXZ4-Firre interaction by DXZ4 deletion does not perturb the $\mathrm{X}$ chromosome inactivation process ${ }^{23}$, a DXZ4 inversion results in altered chromatin interaction profiles along the $\mathrm{Xi}^{26}$.

In this study, we demonstrate that the Firre locus is consistently located at a TAD boundary in multiple species and cell types and we confirm enriched CTCF binding. Based on these features, the Firre locus is an ideal candidate to test the role of CTCF binding in TAD boundary formation. Furthermore, in addition to the role of CTCF, using the Firre lncRNA locus as a model has the unique advantage of allowing us to test whether the presence and expression of a functional lncRNA contribute to TAD boundary formation, as it was recently proposed ${ }^{27-32}$. Thus, we generated Firre deletion and transgenic models in mouse embryonic fibroblasts (MEFs) and embryonic stem cells (mESCs), and performed $\mathrm{Hi}-\mathrm{C}$ in each of the genetically defined cell types, as well as the corresponding wild-type controls. Interestingly, deletion of Firre lncRNA locus, which contains 15 CTCF sites, does not have any effect on TAD boundary formation. Moreover, neither the ectopic insertions of Firre complementary DNA (cDNA) nor its inducible expression lead to disruption of existing, or emergence of novel TAD boundaries. In contrast, the $\mathrm{Xi}$ super-loop interactions were disrupted upon deleting the CTCF

dense Firre locus, thereby proposing a role for Firre in Xi architecture.

Collectively, our results suggest that in addition to CTCF binding, other mechanisms may be required for proper TAD boundary organization.

\section{Results}

Firre harbors dense CTCF binding and strong TAD boundaries. In order to elucidate the contributions of CTCF binding, the genomic insertions, and the expression of a lncRNA to form TAD boundaries, one would ideally interrogate a locus at which all three could be perturbed in parallel. By analyzing publicly available chromatin immunoprecipitation sequencing (ChIP-seq) and $\mathrm{Hi}-\mathrm{C}$ datasets, we determined that the genomic region around the Firre locus harbors one of the highest densities of CTCF binding on chromosome $\mathrm{X}$ across multiple cell types (Fig. 1a,b). On average, the Firre gene body harbors $\sim 15$ CTCF sites and extensive CTCF binding occurs across multiple cell types (Fig. 1a,b). We also find that a TAD boundary is directly located at the Firre locus (hereinafter referred to as "Firre TAD boundary”) (Supplementary Figure 1). This Firre TAD boundary is characterized as one of the strongest TAD boundaries on the X-chromosome (Fig. 1c-f, see Methods), and it is consistently stable across human and mouse cell types. These characteristics of the Firre locus, together with its evolutionary conservation, makes it an optimal model to test the role of CTCF in local TAD structure.

Deletion of Firre leads to depletion of CTCF binding. To determine whether CTCF binding at the Firre locus is necessary for the integrity of the Firre TAD boundary, we generated Firre knockout (KO) MEFs, in which the $\sim 82 \mathrm{~kb}$ deletion encompassed the entire Firre lncRNA locus, as well as the $3^{\prime}$ - and $5^{\prime}$-end (e.g., promoter) regions (Supplementary Figure 2, see Methods). We verified the absence of transcriptional activity of Firre by quantitative reverse-transcriptase PCR (qRT-PCR) (Fig. 2a) and RNA sequencing (RNA-seq) (Fig. 2b). The correlation analysis of the RNA-seq replicates showed high reproducibility (Pearson's correlation $>0.9$ ) in male and female samples (Supplementary Figure 3).

Moreover, we verified the loss of CTCF binding at the deleted Firre locus by performing CTCF ChIP-seq in wild-type and Firre KO female MEFs. As expected, the CTCF binding encompassing the Firre deletion was absent in Firre KO MEFs, whereas ChIPseq profiles of the regions surrounding the deletion were identical to wild-type cells (Fig. 2c). Altogether, we achieved a complete loss of one of the densest CTCF binding regions on the Xchromosome, which allows us to examine their effects on the Firre TAD boundary.

Firre deletion leads to preservation of the TAD boundary. We next assessed whether deletion of the Firre locus impacted the Firre TAD boundary by performing $\mathrm{Hi}-\mathrm{C}$ in male and female wild-type and Firre KO MEFs. Mapping the Hi-C reads to Firre further validates its deletion and the $\mathrm{Hi}$-C interaction heatmaps showed high reproducibility (Fig. 2d,e, Supplementary Figure 4-5, and Supplementary Table 1). Importantly, we identify that the Firre TAD boundary is precisely located within the Firre gene body (Supplementary Figure 7), and resides within the deletion construct (Supplementary Figure 2). To determine whether the effect of CTCF removal to TAD formation is allele specific, we also generated C57BL6/Castaneous (CAST) hybrid MEFs. These hybrid MEFs, which exhibit random $\mathrm{Xi}^{33}$, contain one copy of the C57BL6 allele harboring the Firre KO allele and one copy of the wild-type CAST allele, and thus permit to distinguish between the 
a

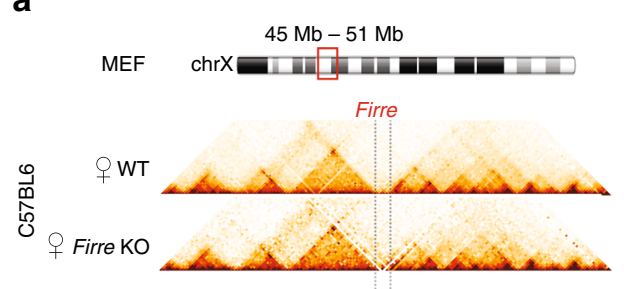

b


d

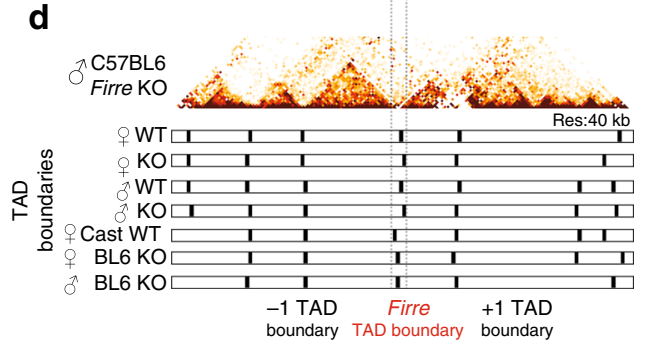

e

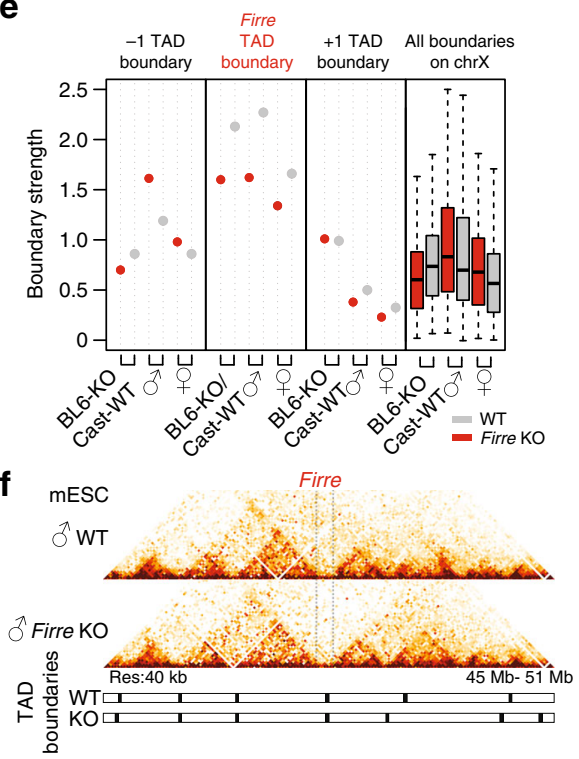

g

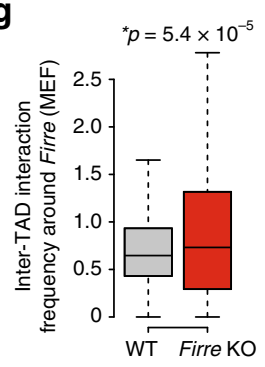

h

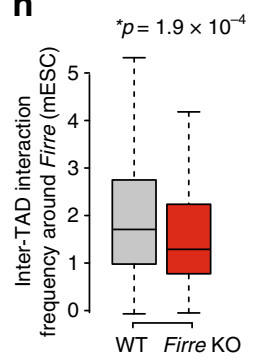

Fig. 3 Firre KO does not result in disruption of TAD boundaries. a-d Hi-C heatmaps showing $\pm 5 \mathrm{Mb}$ of the Firre gene locus (mm9, chr.X: 45-51 Mb) in female wild-type and Firre KO MEFs, (b) male wild-type and Firre KO MEFs, (c) allele-specific haploid chromosomes for female Cast (wild type) and C57BL6 (Firre KO), and (d) male C57BL6 (Firre KO). The TAD boundaries and the insulation plot for each Hi-C dataset is depicted below. e Dot plots showing the boundary strength of the Firre-centered and the neighboring TAD boundaries in wildtype (gray) and Firre KO (red) samples. The TAD insulation scores of all TAD boundaries on chromosome $\mathrm{X}$ is shown as boxplots on the right panel. $\mathbf{f} \mathrm{Hi}-\mathrm{C}$ heatmaps from wild-type (grown on $2 \mathrm{i}$ ) and Firre $\mathrm{KO}$ mouse embryonic stem cells (mESCs) (grown on feeders $+2 \mathrm{i}$ ) showing $\pm 5 \mathrm{Mb}$ of the Firre locus. $\mathbf{g}$, $\mathbf{h}$ Boxplot showing the inter-TAD interaction frequency between the TAD domains neighboring the Firre locus in wild-type (gray) and Firre KO (red) cells (g) in female MEFs and (h) in mESCs. Error bars: s.d. ( ${ }^{*}$-value: Wilcoxon rank-sum test)

parental $\mathrm{KO}$ alleles and examine their individual roles on the Firre TAD boundary formation in female cells.

Remarkably, despite the removal of one of the highest CTCFbinding densities and the complete deletion of the Firre lncRNA on chromosome X (Fig. 1), the TAD boundary is consistently preserved in either male, female, or hybrid MEFs (Fig. 3a-d). Although the insulation scores are decreased at the Firre TAD boundary in the KO samples compared with controls, neither the Firre nor the neighboring TAD boundaries display any significant changes in their insulation profiles (Fig. 3e). To rule out that these results may be cell-type specific, we repeated $\mathrm{Hi}-\mathrm{C}$ in wild-type and Firre $\mathrm{KO}$ male mouse embryonic stem cells (mESCs) grown on feeder cells (Supplementary Figure 6, see Methods). Consistent with the MEF Hi-C results, the Firre TAD boundary is also preserved in Firre KO mESCs. (Fig. 3f). By calculating the interTAD interaction frequency in wild-type and Firre KO MEFs and mESCs, we observe that the TADs surrounding Firre exhibit a higher interaction frequency in the Firre KO MEFs (Fig. 3g), but not in Firre KO mESCs (Fig. 3h), likely to be due to cell-typespecific differences. This finding in MEFs suggests that even though the Firre TAD boundary is preserved, it has been weakened by the genetic deletion, allowing a higher rate of interactions overpassing the boundary in Firre KO MEFs when compared with wild-type controls.
Next, to assess whether the CTCF sites surrounding Firre deletion may act as insulators that could preserve the TAD boundary upon Firre KO, we examined the CTCF ChIP-seq data from female MEFs (Fig. 2), as well as publicly available datasets, for the regions flanking the Firre deletion across several cell types. We identified four peaks flanking the Firre gene body on both sides (Supplementary Figure 7). Of note, these surrounding CTCF peaks are outside of the Firre TAD boundary that is located within Firre gene body (Supplementary Figure 7). By analyzing publicly available ChIP-seq datasets, we observed that $\mathrm{C} 2 \mathrm{C} 12$ cells display reduced binding at the surrounding CTCF sites (Supplementary Figure 8a-c). We reasoned that if the Firre TAD boundary was present in $\mathrm{C} 2 \mathrm{C} 12$ cells with minimal CTCF binding at these sites, it would suggest that the surrounding CTCF sites are not essential for the preservation of the Firre TAD boundary. To determine whether the surrounding CTCF sites are required in establishing the Firre TAD boundary, we performed $\mathrm{Hi}-\mathrm{C}$ in a third cell line: $\mathrm{C} 2 \mathrm{C} 12$ murine muscle cells. We identified a robust TAD boundary formation at the Firre locus in this cell line that displays reduced binding at the surrounding CTCF sites (Supplementary Figure 8a-d). Together, these analyses and experiments suggest that the more variable surrounding CTCF sites can be dispensable for the preservation 
a

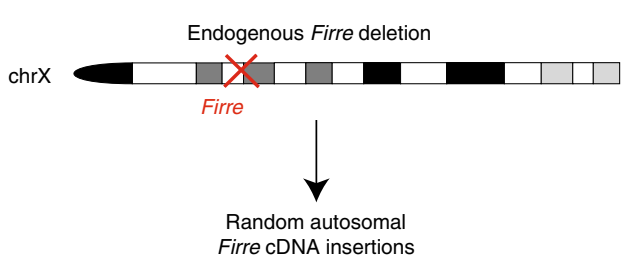

b

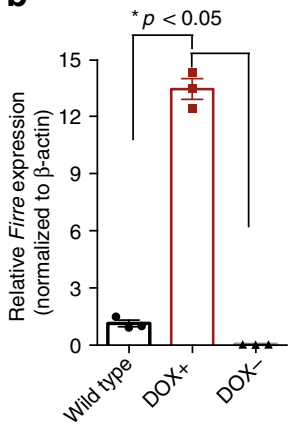

C

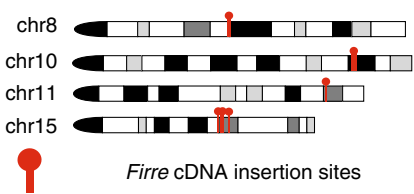

d

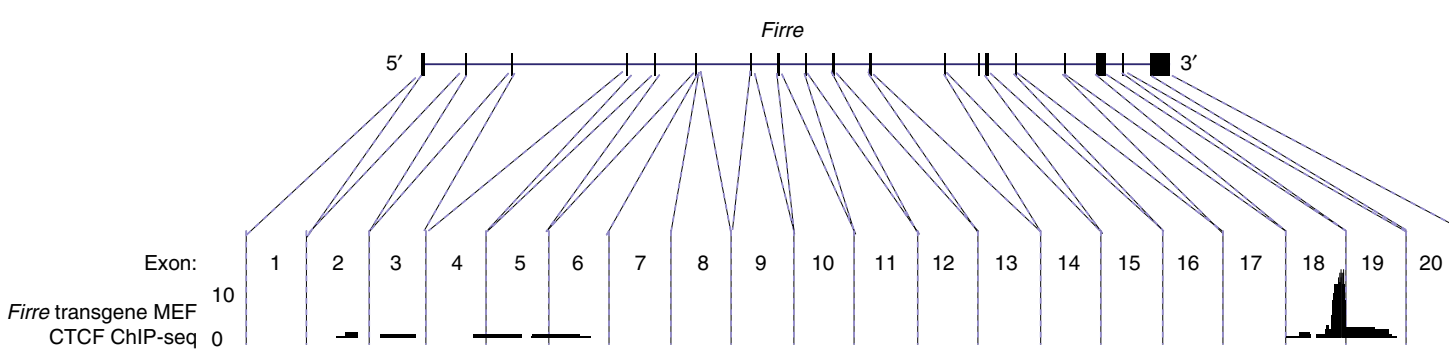

e

$107 \mathrm{Mb}$
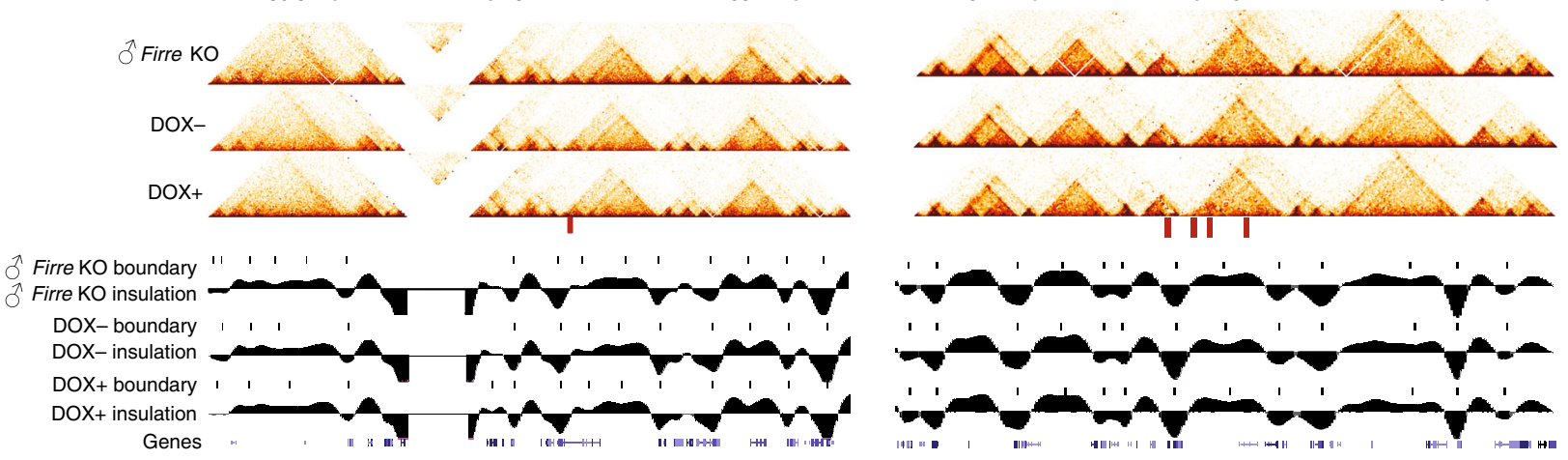

g $\begin{array}{lll}100.5 \mathrm{Mb} & \mathrm{chr} 11 & 106.1 \mathrm{Mb}\end{array}$

h $\quad 63.4 \mathrm{Mb}$
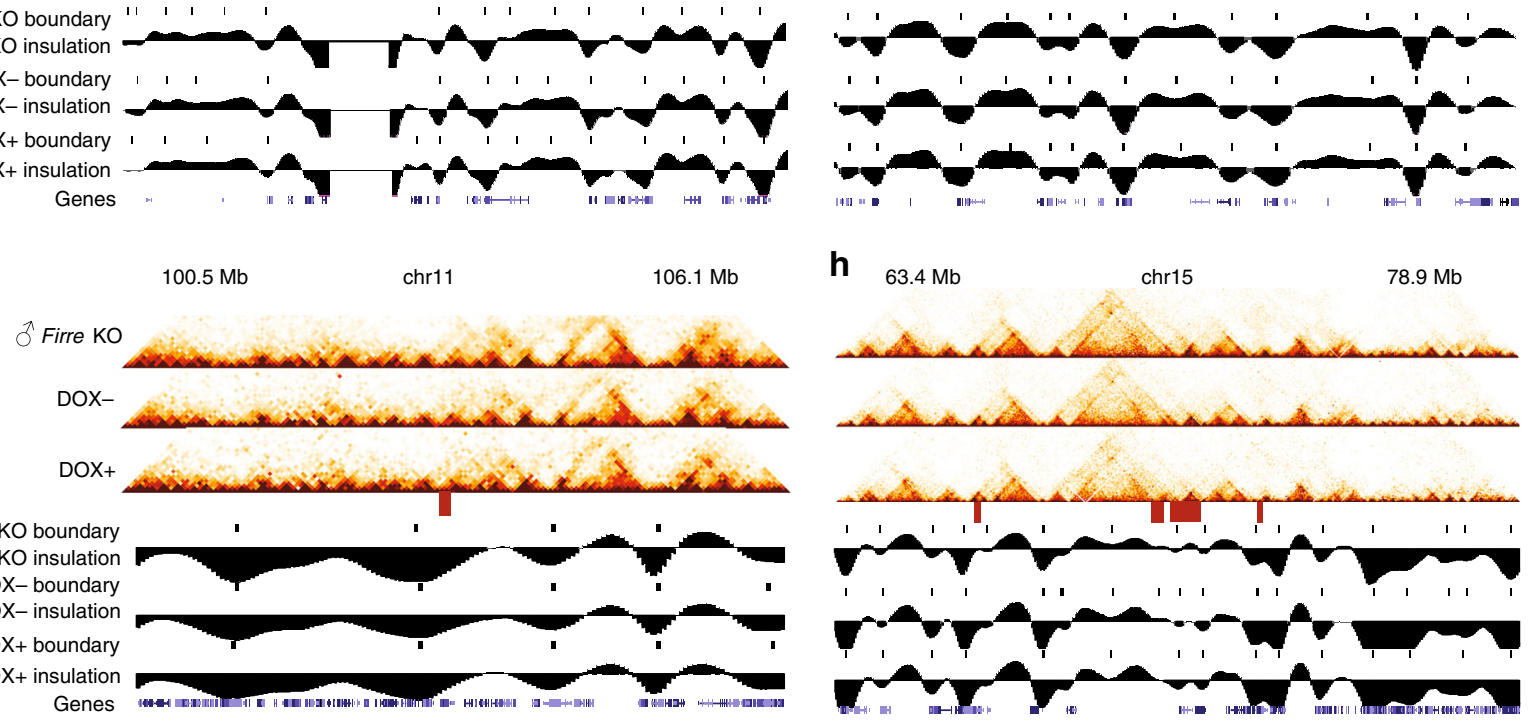

Firre cDNA insertion sites

Fig. 4 TAD boundaries are preserved upon ectopic Firre cDNA insertion and its induced expression at target sites. a Cartoon depicting the approach for the generation of the transgenic MEFs with endogeous Firre KO and ectopic Firre CDNA insertions. $\mathbf{b}$ qRT-PCR showing the induced expression of the Firre cDNA in wild type, DOX ${ }^{-}$, and DOX ${ }^{+}$conditions. Error bars: s.e.m. ( ${ }^{*} p$-value: $t$-test). c Chromosome ideograms showing the Firre $c D N A$ insertion sites on four different chromosomes. d CTCF ChIP-seq signal from DOX ${ }^{-}$transgenic MEFs for each of the exons of Firre cDNA at randomly inserted loci. As the transgenic MEFs harbor an endogenous Firre deletion, the intronic regions did not harbor any ChIP-seq signal. e-h Hi-C heatmaps showing the TAD organization, TAD boundary position, and the insulation plots for male Firre KO, DOX ${ }^{-}$, and DOX samples $\pm \sim 5 \mathrm{Mb}^{+}$of Firre cDNA insertion sites on (e) chr 8, (f) chr 10, (g) chr 11, and (h) chr 15

of the Firre TAD boundary (Supplementary Figure 8d, see Discussion).

Altogether, our findings indicate that the formation and maintenance of the conserved Firre TAD boundary is resilient to (i) removal of CTCF binding, (ii) deletion of the Firre locus, (iii) loss of Firre transcription, and (iv) absence of Firre's functional lncRNA transcript. 
a
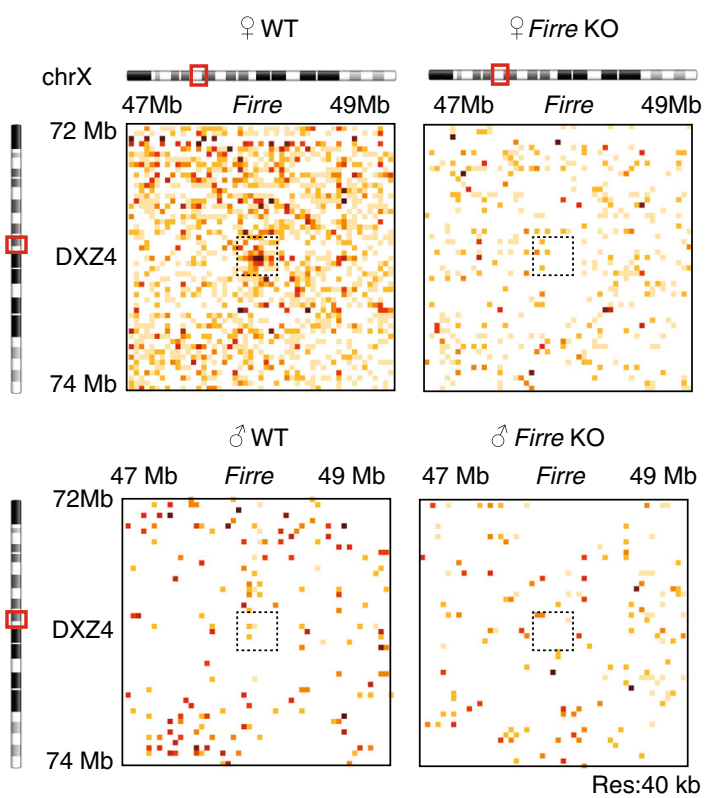

C
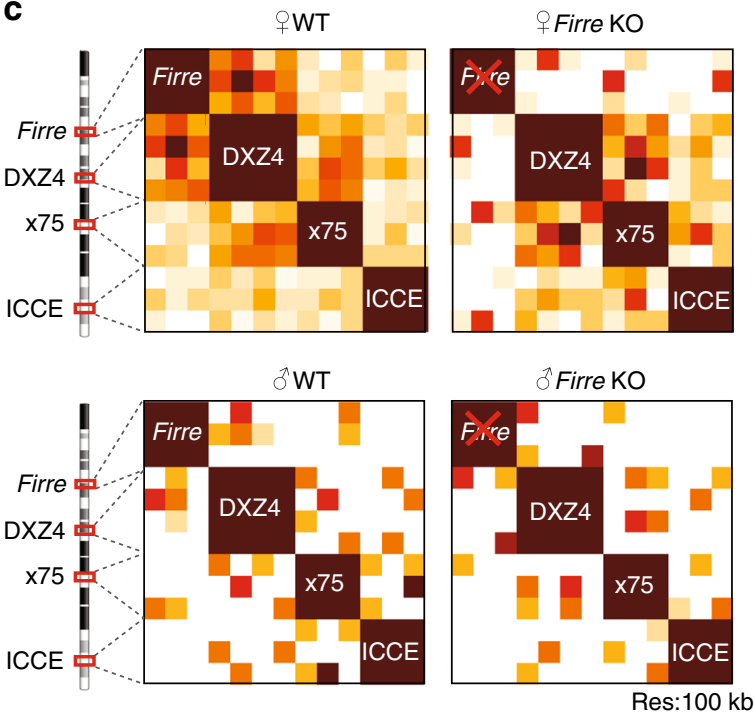

b
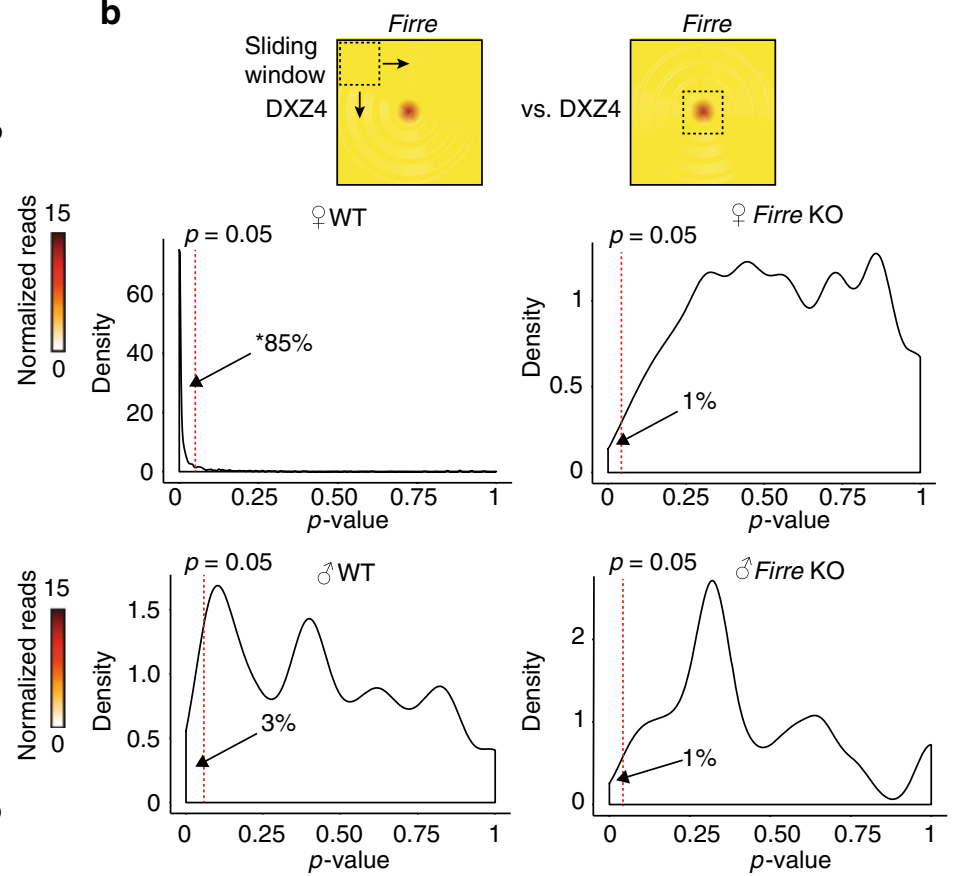

\section{d}
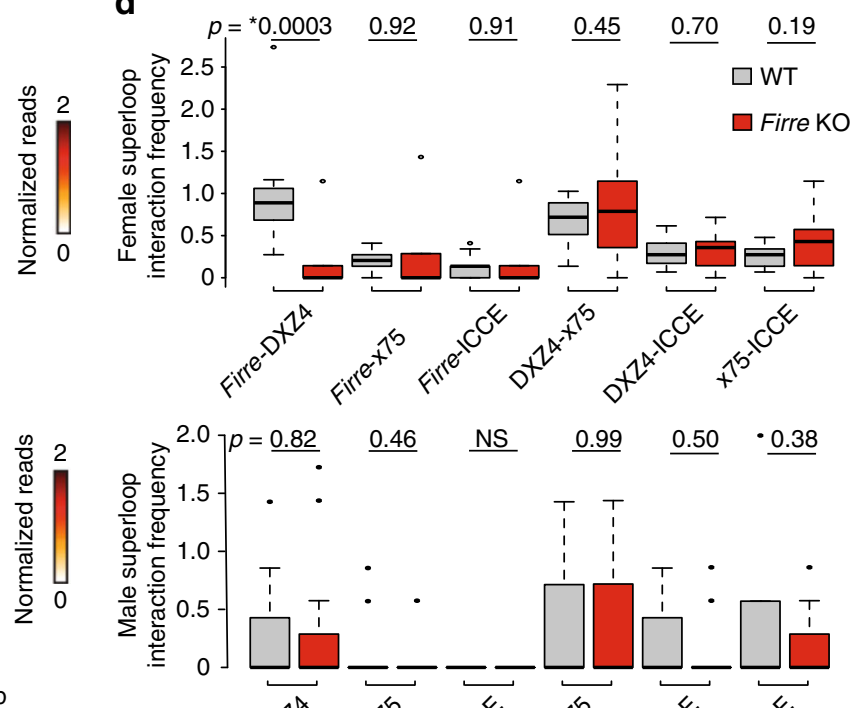

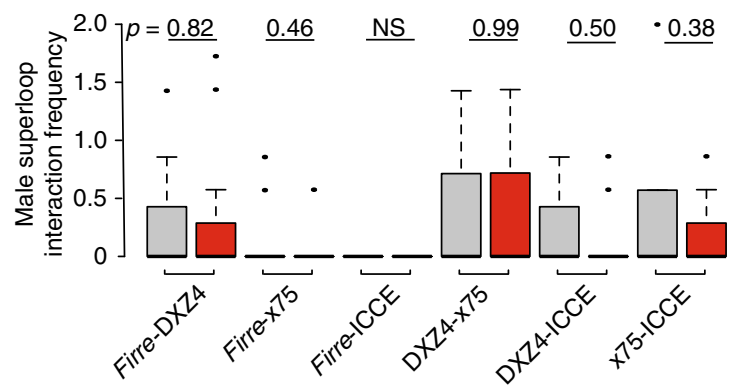

Fig. 5 Firre $\mathrm{KO}$ results in a loss of Firre-DXZ4, and changes in super-loop interactions in female MEFs. a Hi-C heatmaps at $40 \mathrm{~kb}$ resolution in male and female wildtype and Firre KO MEFs showing the interaction frequency between Firre and DXZ4 \pm 1 Mb. $\mathbf{b}$ Forty-two windows of 40 kb bins were slid across the entire heatmap ( $2 \mathrm{Mb} \times 2 \mathrm{Mb}$ in size) to compare the interaction frequency at each position of female and male WT versus KO conditions to the FirreDXZ4 interaction by $t$-tests. The $p$-value distribution in the female or male wild-type samples indicate a drastically enriched significance of Firre-DXZ4 interactions when compared with either the female Firre $\mathrm{KO}$ or male samples $\left({ }^{\star} p<0.05\right.$, one-way ANOVA). c Hi-C heatmap at $100 \mathrm{~kb}$ resolution showing the zoomed-in interactions between the mouse Firre, DXZ4, x75, and ICCE regions $\pm 100 \mathrm{~kb}$ in female and male wild-type and Firre KO MEFs. $\mathbf{d}$ Boxplots showing the interactions among the super-loop regions in wild-type (gray) and Firre KO (red) female (top) and male (bottom) MEF samples. The sample sizes of the boxplots are $n=12$ for Firre-DXZ4, DXZ4-x75, and DXZ4-ICCE interactions, and $n=9$ for all other combinations. $p$-value: $t$-test. Error bars: s.d

Firre cDNA insertions do not alter TAD boundaries. It was previously hypothesized that apart from binding of CTCF, the presence of lncRNAs, or their expression, may be a driving factor to establish TAD boundaries ${ }^{29,32}$. Therefore, although the deletion of the CTCF-rich Firre locus is not sufficient to disrupt TAD boundary formation, we sought to determine whether an ectopically inserted Firre cDNA would establish novel TAD boundaries, either in the presence or absence of its transcription. To do this, we inserted Firre cDNA under the regulation of a doxycycline (DOX)-inducible promoter into random genomic sites in male MEFs that harbor one active $\mathrm{X}$ chromosome, but with an endogenous Firre deletion (Fig. 4a). Upon DOX induction (DOX ${ }^{+}$), we measured a $\sim 13$-fold increase of Firre cDNA expression from the ectopic loci by qRT-PCR, confirming the induced expression of the ectopically inserted Firre cDNA (Fig. 4b). We then performed $\mathrm{Hi}-\mathrm{C}$ on non-induced $\left(\mathrm{DOX}^{-}\right)$and induced 
a
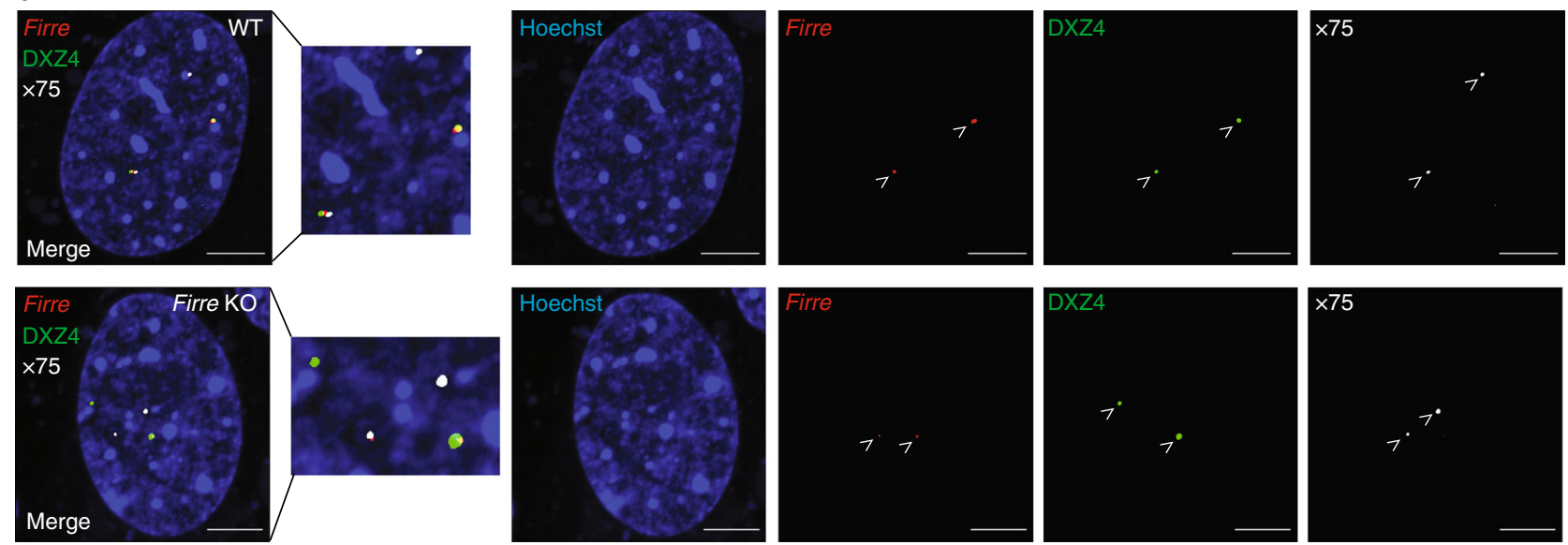

b


Fig. 6 CRISPR live-cell imaging and 3C validates the changes in super-loop interactions. a Four-color CRISPR live-cell imaging (CLING) from female wildtype and Firre KO MEFs. Firre (red), DXZ4 (green), and x75 (white) loci were simultaneously visualized with Hoechst staining (blue). Pseudo-coloring was used for visual simplicity. Scale bar: $5 \mu \mathrm{m}$. b Quantification of the colocalization percentages between Firre-DXZ4, DXZ4-x75, and Firre-x75 between wildtype (black) and Firre KO (red) MEFs. ( ${ }^{*} p$-value: $\chi^{2}$-test, $n>80$ nuclei). Error bars: s.e.m. c Chromosome conformation capture (3C) analysis showing the interaction frequency ratios of Firre-DXZ4, DXZ4-x75, and Firre-x75 in female Firre KO vs. wild-type MEFs ( ${ }^{\star} p$-value: $t$-test, $n=3$ ). The black arc indicates $3 C$ enrichment in the wild-type samples, whereas the red arc represents enrichment in the Firre KO samples

$\left(\mathrm{DOX}^{+}\right)$transgenic MEFs, and identified four chromosomes with multiple Firre insertions (Fig. 4c, Methods). To determine whether the inserted Firre cDNA can recruit CTCF, we performed CTCF ChIP-seq on DOX $^{-}$transgenic MEFs and found CTCF binding on exon 18 on the Firre cDNA (Fig. 4d, introns and intronic CTCF binding were excluded).

We next tested whether the local TAD boundaries at the ectopic insertion sites were altered in any way by the presence of the Firre cDNA or ectopic CTCF binding. By comparing the $\mathrm{DOX}^{-}$transgenic Firre MEFs with male Firre KO MEFs, we found that none of the ectopic Firre insertions, despite active CTCF binding, significantly altered the local genomic structure or created new TAD boundaries (Fig. $4 \mathrm{e}-\mathrm{h}$ ). We then tested whether the transcriptional activation of Firre at these ectopic loci would have an impact on TAD organization. To do this, we performed $\mathrm{Hi}-\mathrm{C}$ on $\mathrm{DOX}^{+}$transgenic MEFs, and compared the TAD boundaries with $\mathrm{Hi}-\mathrm{C}$ data of $\mathrm{DOX}^{-}$transgenic MEFs. We observed that the presence of transcription at the Firre insertion sites did not affect TAD boundary formation (Fig. 4e-h). Together, these data suggest that neither ectopically inserted CTCF sites nor the ectopic genomic insertion of a cDNA, nor the act of transcription at the ectopically inserted loci are sufficient to alter endogenous TAD boundaries or create novel TADs at target sites.
Firre deletion results in loss of Firre-DXZ4 interactions. X chromosome inactivation is a critical biological process that involves massive reorganization of chromosome $\mathrm{X}$ with loss of local structures and formation of transcriptionally silent megadomains ${ }^{12,21-25}$. The DXZ4 macrosatellite, which is located at the hinge point of two mega-domains, has been shown to interact with the Firre locus ${ }^{23}$. We therefore investigated the role of Firre in this interaction by $\mathrm{Hi}-\mathrm{C}$. In female primary MEFs, we confirmed the previous finding that Firre strongly interacted with DXZ4. However, this interaction was abolished in female Firre KO MEFs (Fig. 5a). In contrast, Firre did not show a specific association with DXZ4 in male MEFs, in which the only X chromosome is active (Fig. 5a). To validate the specificity of the Firre-DXZ4 interaction, we selected a window on the heatmap $(6 \times 7=4240 \mathrm{~kb}$ bins $)$ and slid it bin-by-bin across the entire heatmap ( $2 \mathrm{Mb} \times 2 \mathrm{Mb}$ in size). We then compared the interaction frequency of the sliding window at each position with the detected Firre-DXZ4 interaction (same window size) via a $t$-test. We performed this analysis for each condition (female and male wildtype and Firre KO). By plotting the density of the $p$-values ( $t$ test), we observed in the female wild-type dataset that the FirreDXZ4 interaction is significantly enriched compared with the female Firre $\mathrm{KO}$ and the male samples $(p<0.05$, one-way analysis of variance). 
In human cells, the interactions of Firre on $\mathrm{Xi}$ have been reported to be associated with the formation of a super-loop involving the DXZ4 macrosatellite, x75, and ICCE regions, all of which are lncRNA loci ${ }^{23}$. To investigate whether such a superloop might occur in mice, and to determine if Firre is required to form any such structure, we investigated the interactions between mouse Firre, DXZ4, x75, and ICCE regions (Fig. 5c). Hi-C in female Firre KO MEFs showed that Firre deletion disrupted its association with DXZ4 (Fig. 5c,d). In contrast, male wildtype and KO MEFs did not display any notable changes (Fig. $5 c, d$ ). These results suggest an architectural role of Firre in the Xi super-loop formation.

To validate the loss of Firre-DXZ4-x75 interactions, we performed four-color CRISPR/Cas9 live-cell imaging (CLING) in female wild-type and Firre KO MEFs ${ }^{34}$. To do this, we used a nuclease-null mutant of the Streptococcus pyogenes Cas9 protein (dCas9) and pools of three single-guide RNAs (sgRNAs) separately targeting Firre, DXZ4, and x75 regions (Supplementary Table 4). The sgRNAs targeting Firre and DXZ4 are internally appended with three copies of MS2 and PP7 motifs, respectively, and the 755 sgRNAs with six copies of Puf1 RNA-aptamer motifs. Co-transfection of corresponding RNA-binding proteins (MS2, PP7, or Pum1) fused to a fluorescent proteins (mVenus, mCherry, or iRFP670), in combination with nuclear Hoechst staining, has enabled us to simultaneously visualize Firre, DXZ4, and $x 75$ loci in living cells ${ }^{34}$. The sgRNAs targeting the Firre locus were designed within $650 \mathrm{bp}$ adjacent to the Firre deletion $\left(5^{\prime}\right.$ end), so that both the wild-type and Firre KO loci could be visualized. When we quantified the colocalization frequencies, we detected a significant reduction between Firre and DXZ4 in Firre KO cells, when compared with wild-type controls $\left(p=8.7 \times 10^{-7}\right.$, $\chi^{2}$-test, Fig. 6a,b). In contrast, the Firre KO MEFs displayed a modest, nonsignificant colocalization increase for the DXZ4-x75 interaction $\left(p=0.102, \chi^{2}\right.$-test) when compared with wild-type MEFs. Of note, we observed a significantly increased colocalization of Firre with the $\mathrm{x} 75$ region in KO MEFs $\left(p=0.008, \chi^{2}\right.$-test, Fig. 6a,b).

To further verify the changes in the super-loop formation in the Firre KO cells, we performed chromosome conformation capture $^{35}$ (3C) in female wild-type and Firre KO MEFs. We confirmed the significant loss of Firre-DXZ4 ( $p=0.02, t$-test) in the Firre KO MEFs when compared with wild-type MEFs (Fig. 6c and Supplementary Figure 9). The difference between the DXZ4x75 3C interaction frequency correlated with $\mathrm{Hi}-\mathrm{C}$ and live-cell imaging, although it was not significant $(p=0.75, t$-test $)$. Consistent with the live-cell imaging results, the Firre-x75 3C interaction frequency was also significantly increased $(p=0.01, t$ test) in the Firre KO sample (Fig. 6c). Taken together, through $\mathrm{Hi}-\mathrm{C}, 3 \mathrm{C}$, and CLING experiments, we conclude that the Firre locus and/or its lncRNA product are important in regulating super-loop interactions in female cells.

\section{Discussion}

The genome harbors a non-random three-dimensional conformation that is dynamically reconfigured. The organization of one of the fundamental units, TAD structures, is strongly associated with directional binding of $\mathrm{CTCF}^{8}$. The current model explaining the TAD boundaries, known as the loop extrusion model, suggests that loop extruding factors such as cohesin and condensin, slide along the chromatin and protrude the DNA until two convergent CTCF sites are reached ${ }^{8,14,36}$, therefore implicating CTCF as one of the important organizers of chromatin $^{7,13,37}$. However, discrepancies have been reported in terms of the requirement of deleting CTCF-binding sites, or depleting CTCF to disrupt TAD boundaries $9,11,16,38,39$.
In this study, we genetically removed the evolutionary conserved Firre locus that has both one of the highest CTCF densities in the genome across and a stable TAD boundary across many cell types and species. Interestingly, the Firre KO did not lead to perturbation of the TAD boundary, but led to a decrease in the strength of the Firre TAD boundary in MEFs, suggesting that $\mathrm{TAD}$ organization at the Firre locus may not be individually dependent on CTCF binding or Firre lncRNA expression. Although not directly located at the Firre TAD boundary (Supplementary Figure 7), additional flanking CTCF sites outside of the Firre deletion may compensate for the loss of CTCF binding at this locus and keep Firre's TAD boundary formation intact in Firre KO cells. In addition, we further show that, despite the recruitment of CTCF, the ectopic insertion or the induced expression of the Firre cDNA at ectopic sites are not sufficient to alter the TAD landscape of local chromatin environment. These results suggest that the establishment of TADs may be mediated by CTCF-independent, multi-layered mechanisms, that are likely to be redundant to preserve the robustness of TAD structures. The current mechanistic model for intra-chromosomal chromatin organization is the loop extrusion model ${ }^{7,13,37}$. Consistent with this model, the inversion of CTCF sites abrogates chromatin looping interactions ${ }^{8,36}$, and the depletion of CTCF results in the weakening of TAD boundaries ${ }^{16}$. In addition, cohesin removal also leads to the loss of TADs ${ }^{40,41}$. The Firre locus, which is bound by CTCF, is also extensively bound by cohesin, as well as the transcription factor YY1, which was identified to associate with $\mathrm{CTCF}^{42}$ and was implicated as a structural regulator of looping interactions ${ }^{43}$. The preservation of the Firre TAD boundary in our deletion model therefore suggest that these two factors may also harbor redundant functions.

We therefore conclude that, in addition to the well-established mechanism of convergent CTCF sites being the primary factor for loop extrusion, alternative mechanisms may also be responsible for the establishment of TADs. Several recent studies have provided support to this hypothesis $8,44,45$.

In addition to convergent CTCF sites, there are other aspects of chromatin which can mediate TAD boundary formation. It has been shown that even though $\sim 75 \%$ of all looping interactions can be explained by convergent CTCF orientations, the remaining $\sim 25 \%$ of interactions were bound by CTCF with forward-forward, reverse-reverse, and divergently oriented CTCF motifs ${ }^{8}$. In another report, $\sim 10 \%$ of all the CTCF motifs at the anchor of loops did not obey the convergent rule ${ }^{45}$. Interestingly, the folding of Drosophila contact domains was found to be independent of CTCF orientation ${ }^{44}$, suggesting the role of alternative architectural proteins in chromosome structure. Consistent with this, in human cells, FIRRE appears to form a circular structure with divergently oriented CTCF sites, whereas this circular structure is not prevalent in mouse (Supplementary Figure 10-11). Importantly, in the mouse genome, the CTCF orientations at the $3^{\prime}$-end of Firre gene and the neighboring boundary (the -1 TAD boundary in Fig. 3a) show forward-forward CTCF motif orientations, suggesting the presence of interactions with non-convergent CTCF-binding motifs (Supplementary Figure 11).

There is further evidence in the literature for CTCFindependent mechanisms of TAD boundary formation. For instance, gene activation and repression was shown to have important roles in genome folding independent of $\mathrm{CTCF}^{44}$. Additional studies have also highlighted the role of chromatin condensation and de-condensation via topoisomerases and chromatin remodeling factors to organize the TAD structures $^{46-50}$, and the stiffness of chromatin has been postulated to mediate TAD boundary formation ${ }^{51}$. Altogether, our findings 
therefore provide experimental insight into the redundancy of CTCF in TAD boundary organization, in the context of the Firre locus.

Through Hi-C, CLING, and 3C, we furthermore show that the deletion of Firre results in the perturbation of super-loop interactions, specifically with the DXZ4 macrosatellite (Figs. 5,6). Interestingly, in female Firre KO MEFs, via CLING and 3C, we identified an increased interaction frequency between the region surrounding Firre and $\mathrm{x} 75$, suggesting that apart from the lncRNA genes in these loci, other features of chromatin may also be involved in mediating the super-loop formation. Future studies will determine the functional significance of the disruptions of the super-loop formation on X chromosome inactivation.

In summary, our study suggests the redundancy of CTCF binding, local lncRNA, and its transcription in the establishment of TADs, and sheds new light on an architectural role for Firre in higher order X chromosome architecture.

\section{Methods}

Publicly available Hi-C and CTCF ChIP-seq analysis. Hi-C interaction matrices for K562 (female), HMEC (male), NHEK (unisex), sperm (male), Patski fibroblasts (female), and mouse neuronal stem cells (male) were downloaded using the Juicebox software $^{12,52,53}$, and the heatmaps were generated with the publicly available script "heatmap.pl" available through Github (https://github.com/dekkerlab/ cworld-dekker). The RPE-1 (female) and mESC Hi-C datasets ${ }^{2,23}$ were downloaded and analyzed with the HiC-Pro package v2.7.8 ${ }^{54}$. The CTCF ChIP-seq datasets for human RPE-1 cells and mouse sperm cells were downloaded from previously published studies ${ }^{55,56}$. All the other CTCF datasets were downloaded from the ENCODE project database ${ }^{57}$. To identify the CTCF enrichment of genomic loci, we calculated the number of CTCF peaks for each $130 \mathrm{~kb}$ and $80 \mathrm{~kb}$ sliding window on chromosome $\mathrm{X}$, for human and the mouse genomes, respectively.

Preparation of Firre KO cells. The Firre KO mouse was generated by inserting a neomycin cassette flanked by loxP sites at position mm9:chrX:47908463-47908464, and by inserting a hygromycin cassette flanked by loxP sites at $\mathrm{mm} 9$ :

chrX:47990293-47990294. Recombination between the loxP sites resulted in a $~ 82$ kb deletion around the Firre locus. A tet-inducible Firre overexpression mouse was generated by cloning a mouse isoform of Firre into a modified pTRE2 vector that lacks the beta globin intron and the construct was microinjected into C57BL6/J-129 F1 hybrid embryos in order to generate transgenic mice. MEFs were prepared at E13.5. Embryos were collected in $1 \times$ phosphate-buffered saline (PBS) and individual embryos were eviscerated and the head, forelimbs, and hind limbs were removed. Individual embryo caucuses were then placed into $6 \mathrm{~cm}^{2}$ tissue culture plate and $1 \mathrm{~mL}$ of pre-warmed $37^{\circ} \mathrm{C}$ TrypLE (Thermo Fisher 12604013) was added to each well and incubated for $20 \mathrm{~min}$ at $37^{\circ} \mathrm{C}$. Embryos were dissociated using a P1000 tip with gently pipetting and then MEF media was added. Cells were cultured for 5-7 days and cryostocks of individual lines were generated. Subsequent experiments were performed from thaws from the cryostocks ${ }^{58}$. Firre transgenic female mice were mated to CAGs-rtTA3 males (Jackson Lab 016532) and individual embryos at E13.5 were collected and used to prepare MEF lines. Firre wildtype and KO MEFs were also prepared from E13.5 embryos. MEFs were genotyped for the sex-specific region (Sry), Firre wild-type, Firre KO, Firre transgenic, and rtTA3 alleles (Supplementary Table 2). MEFs were used up to passage 4. MEFs were cultured in $1 \times$ Dulbecco's modified Eagle's medium (DMEM) (Invitrogen 11965-118), fetal bovine sSerum (Gibco 10082139), L-glutamine (ThermoFisher 25030081), and penicillin/streptomycin (ThermoFisher 15140122). The DOX induction was performed by treating the cells with $2 \mu \mathrm{g} / \mathrm{mL}$ DOX (Sigma D9891) for $72 \mathrm{~h}$. The media was changed every $48 \mathrm{~h}$.

Wild-type C2C12 cells were obtained from ATCC (CRL-1772) and were cultured by using the same conditions as MEFs described above.

ESCs were derived on MEF, Lif, and one inhibitor (GSK inhibitor) ES media with serum replacement. Freeze downs were at passage 2 from a 24 -well plate. The wild-type N3 male mESCs were cultured with $2 \mathrm{i}$ media. The Firre KO mESCs were co-cultured with irradiated feeder MEFs (ATCC, SCRC-1040.1) with 2i media. Feeder depletion was performed by plating disassociated cells on a gelatinized plate for $20 \mathrm{~min}$ at $37^{\circ} \mathrm{C}$ followed by reseeding the supernatant containing the mESCs to a new gelatinized plate. This procedure was repeated two consecutive times. The animal protocols in this study have been approved by Institutional Animal Care and Use Committee (IACUC) and Harvard University (11-13-1).

qRT-PCR analysis. RNA was extracted by using TRIzol Reagent (ThermoFisher 15596018) and cDNA was generated by using the SuperScript III Reverse Transcriptase kit (ThermoFisher 18080044), according to manufacturer's instructions qRT-PCR was performed by using the primers listed in Supplementary Table 2. The qRT-PCR data was analyzed by using the $2^{(- \text {delta/delta C } t)}$ method.
RNA sequencing. Total RNA was extracted from wild-type and Firre KO MEFs using TRIzol followed by RNeasy Mini Qiagen extraction kit according to manufacturer's protocol. RNA-seq libraries were generated using the Illumina TruSeq (version 2) kit and sequenced on Illumina HiSeq2500 instrument. RNA-seq reads were aligned with RNA-seq analysis performed by filtering and mapping the reads by Bowtie $2^{59}$, quantifying the transcripts by RSEM v1.2.2960. Differential gene expression was calculated using the Deseq2 version 1.4 .5 package in $\mathrm{R} 3.1 .0$ using the mean value of gene-wise dispersion estimates ${ }^{61}$. To find significant differentially expressed genes, we used $<0.01$ for adjusted $p$-value and $>1 \log 2$ fold change.

ChIP-seq analysis. The ChIP assay was performed by first crosslinking $\sim 10$ million cells with $1 \%$ formaldehyde at room temperature for $10 \mathrm{~min}$, washing the cells twice with $1 \times \mathrm{PBS}$, and then followed by lysing the cells with $1 \mathrm{~mL}$ of Lysis Buffer A (50 mM HEPES, $140 \mathrm{nM} \mathrm{NaCl}, 1 \mathrm{mM}$ EDTA pH 8.0, 10\% Glycerol (Sigma G5150), 0.5\% NP-40 (Igepal CA-630, Sigma I3021), 0.25\% Triton X-100), centrifuging the cells at $1,350 \times g$ for $5 \mathrm{~min}$ at $4{ }^{\circ} \mathrm{C}$, and re-suspending the pellet with 1 $\mathrm{mL}$ of Lysis Buffer B (10 mM Tris-HCl pH 8.0, $200 \mathrm{nM} \mathrm{NaCl}, 1$ mM EDTA pH 8.0, $1 \mathrm{mM}$ EGTA), centrifuging the cells at $1350 \times \mathrm{g}$ for $5 \mathrm{~min}$ at $4{ }^{\circ} \mathrm{C}$, and a final resuspension of the pellet with $300 \mu \mathrm{L}$ of Lysis Buffer C $(10 \mathrm{mM}$ Tris- $\mathrm{HCl}, \mathrm{pH} 8.0$ $100 \mathrm{mM} \mathrm{NaCl}, 1 \mathrm{mM}$ EDTA pH 8.0, $1 \mathrm{mM}$ EGTA, 0.1\% Sodium deoxycholate (Sigma D6750), and $0.5 \% \mathrm{~N}$-lauroylsarcosine (Sigma L5777) ${ }^{62}$. All the lysis buffers included the cOmplete, Mini Protease Inhibitor Cocktail (Sigma 11836153001). The chromatin was sheared by using a Covaris S220 instrument. The pull-down was performed using $10 \mu \mathrm{g}$ of CTCF antibody (1:1000 dilution, Millipore Sigma 07-729). The reads were aligned to the mm9 human genome using the Bowtie2 tool $^{59}$. The ChIP-seq data was analyzed using the HOMER suite ${ }^{63}$. The motif orientations were determined by the FIMO software ${ }^{64}$, using the CTCF motif position weight matrix (MA0139.1) from the JASPAR database ${ }^{65}$

Generation of Hi-C libraries. Hi-C libraries were generated with an in-situ ligation protocol using the HindIII restriction enzyme ${ }^{66}$, Briefly, $\sim 25$ million cells were crosslinked with $1 \%$ formaldehyde for 10 minutes at room temperature. Then, the chromatin was digested with HindIII, end-labeled with biotin-14-dCTP (Thermo Fisher 19519016), and in-situ ligation was performed. Following phenol-chloroform extraction, the biotin was removed from unligated ends and the DNA was sheared by using a Covaris S220 instrument. After A-tailing, biotin pull-down, and adapter ligation, paired-end sequencing was performed on a HiSeq instrument. Each Hi-C library was generated in at least two biological replicates from MEFs prepared from at least two distinct mouse embryos, with the exception of transgenic MEFs and mESCs, which were prepared in technical duplicates.

Analysis of Hi-C datasets. $\mathrm{Hi}-\mathrm{C}$ mapping, filtering, correction, and binning was performed with the HiC-Pro software v2.7.8 ${ }^{54}$. The reads were mapped to the $\mathrm{mm} 9$ mouse reference genome. For allele-specific $\mathrm{Hi}-\mathrm{C}$ analysis, a high-quality singlenucleotide polymorphism (SNP) list for the C57BL6NJ and CastEiJ genomes was generated by using the $\mathrm{mm} 9$ annotation from the Mouse Sanger Database ${ }^{67}$, using the HiC-Pro "extract_snps.py" tool. Next, C57BL6NJ/CastEiJ SNP-masked mm9 reference genome was generated using the bedtools "maskfasta" tool ${ }^{68}$. Then, allele-specific Hi-C data was analyzed using the HiC-Pro "ALLELE_SPECIFIC_SNP" configuration option ${ }^{54}$. Supplementary Table 1 lists the paired-end read counts for each biological replicate of the $\mathrm{Hi}-\mathrm{C}$ datasets. There was a high correlation among all the $\mathrm{Hi}-\mathrm{C}$ biological replicates, indicating the high quality and reproducibility of the datasets. Therefore, we pooled all biological replicates for each condition and mapped, filtered, corrected and binned them as a single $\mathrm{Hi}-\mathrm{C}$ dataset and used the pooled datasets for all subsequent analyses. For the $\mathrm{Hi}-\mathrm{C}$ analysis of endogenous Firre KO with ectopic Firre cDNA insertion MEFs, the Hi$\mathrm{C}$ reads were mapped to a custom $\mathrm{mm} 9$ genome with an extra chromosome consisting of the insertion cassette (TRE element, cytomegalovirus promoter, Firre cDNA, and polyA terminator). Then, "inter-chromosomal interactions" between the Firre custom chromosome and all the mouse chromosomes were plotted in the $\mathrm{DOX}^{-}$and $\mathrm{DOX}^{+} \mathrm{Hi}-\mathrm{C}$ datasets. The regions that displayed consistent "interactions" with the Firre custom chromosome in $\mathrm{DOX}^{-}$and $\mathrm{DOX}^{+}$samples were determined as Firre cDNA insertion sites. For the super-loop analysis in Fig. 5, the human $x 75$ and ICCE coordinates ${ }^{23}$ were lifted over to the $\mathrm{mm} 9$ genome. The center line of all boxplots represent the median, the whiskers represent the maximum and minimum values, and the upper and lower bounds of the box represent the top first and third quantiles of the datasets.

Insulation and TAD Boundary Analysis. TAD analysis was performed with the "Insulation Method"69. A publicly available script (matrix2insulation.pl) was used to detect the TAD boundaries, with the following options: "-is 480000 -ids 320000 -im iqrMean -nt 0 -ss 160000 -yb 1.5 -bmoe 0 -bg”. The script can be accessed through GitHub (https://github.com/dekkerlab/cworld-dekker).

Chromosome conformation capture. $3 \mathrm{C}$ assay was performed by using the HindIII enzyme ${ }^{35}$, with the modification that in-situ ligation was performed ${ }^{66}$ MEFs were fixed with $1 \%$ formaldehyde in serum-free a-MEM for $10 \mathrm{~min}$ at room temperature. Formaldehyde was quenched by the addition of $0.125 \mathrm{M}$ glycine. Nuclei were released by dounce homogenization in ice-cold lysis buffer $(10 \mathrm{mM}$ 
Tris- $\mathrm{HCl} \mathrm{pH} \mathrm{8.0,10} \mathrm{mM} \mathrm{NaCl,} \mathrm{0.2 \%} \mathrm{NP-40)} \mathrm{containing} \mathrm{cOmplete,} \mathrm{Mini} \mathrm{Protease}$ Inhibitor Cocktail (Sigma 11836153001). Nuclei were collected and subjected to overnight digestion at $37^{\circ} \mathrm{C}$ with $400 \mathrm{U}$ of HindIII (NEB R0104L). Then, in-situ ligation was performed for $4 \mathrm{~h}$ at $16^{\circ} \mathrm{C}^{66}$. The crosslinks were reversed by incubating the samples at $65^{\circ} \mathrm{C}$ overnight in the presence of proteinase $\mathrm{K}$, and the DNA was purified by phenol-chloroform extraction. Supplementary Table 3 lists the $3 \mathrm{C}$ primers used in this study. The $3 \mathrm{C}$ libraries were prepared in three biological triplicates, and the $3 \mathrm{C}$ amplicons from each replicate were quantified in three technical replicates. The sample to sample variation was normalized by using the Gapdh region as a control locus. The results in Fig. 6 represent the 3C interaction frequency ratios of Firre $\mathrm{KO} /$ wildtype values. All $3 \mathrm{C}$ products were analyzed on a $2 \%$ agarose gel stained with ethidium bromide. Gel quantifications were performed with the Adobe Photoshop ${ }^{\circledR}$ software.

CRISPR live-cell imaging. Three specific sgRNAs for each tested locus were designed using the Broad Institute sgRNA Design Tool (http://www.broadinstitute. org/rnai/public/analysis-tools/sgrna-design-v1 $)^{70}$ and were cloned into vectors expressing either three MS2, three PP7 or six Puf1 motifs (Addgene 68426, 68424) ${ }^{71}$. The sgRNA sequences are listed in Supplementary Table 4. Next, using Lipofectamine 3000 (ThermoFisher Scientific, L3000008), pools of the three sgRNAs for each targeted locus ( $375 \mathrm{ng}$ ), dCas9 (Addgene 68416, $625 \mathrm{ng}$ ), and vectors expressing the corresponding RNA-binding proteins fused to fluorescent proteins (MS2-mVenus, PP7-mCherry, Pum1-iRFP670, each $500 \mathrm{ng}$ ) were transfected into MEFs and plated on LabTek vl glass chamber slides. After $48 \mathrm{~h}$ of incubation, FluoroBrite $^{\text {tw }}$ DMEM Media (ThermoFisher Scientific, A1896701), and 1 drop NucBlue $^{\circledast}$ Live ReadyProbes ${ }^{\circledast}$ Reagent (ThermoFisher Scientific R37605) were added to stain the nuclei. Images were acquired from more than 80 live cells ( 95 for wild type, 84 for Firre KO cells) by using the LSM880 with Airyscan (Zeiss) microscope, equipped with the oil immersion objective Plan-Apochromat $\times 63 / 1.4$ oil DIC M27 at the Harvard Center for Biological Imaging (HCBI). Raw images were processed in ZEN (blue edition, Zeiss) and colocalization was assigned when either merged or overlapping signals $(<50 \mathrm{~nm}$ distance) occurred. In contrast, distinctly separated signals ( $>50 \mathrm{~nm}$ distance) indicated no colocalization.

Data availability. Sequencing data have been deposited in the Gene Expression Omnibus under the accession number GSE98632. All other data are available from the corresponding authors upon reasonable request.

Received: 8 December 2017 Accepted: 27 February 2018

Published online: 13 April 2018

\section{References}

1. Nora, E. P. et al. Spatial partitioning of the regulatory landscape of the Xinactivation centre. Nature 485, 381-385 (2012).

2. Dixon, J. R. et al. Topological domains in mammalian genomes identified by analysis of chromatin interactions. Nature 485, 376-380 (2012)

3. Grubert, F. et al. Genetic control of chromatin states in humans involves local and distal chromosomal interactions. Cell 162, 1051-1065 (2015).

4. Pope, B. D. et al. Topologically associating domains are stable units of replication-timing regulation. Nature 515, 402-405 (2014).

5. Norton, H. K. \& Phillips-Cremins, J. E. Crossed wires: 3D genome misfolding in human disease. J. Cell. Biol. 216, 3441-3452 (2017).

6. Phillips, J. E. \& Corces, V. G. CTCF: master weaver of the genome. Cell 137, 1194-1211 (2009).

7. Fudenberg, G. et al. Formation of chromosomal domains by loop extrusion Cell Rep. 15, 2038-2049 (2016)

8. Guo, Y. et al. CRISPR inversion of CTCF sites alters genome topology and enhancer/promoter function. Cell 162, 900-910 (2015).

9. Lupianez, D. G. et al. Disruptions of topological chromatin domains cause pathogenic rewiring of gene-enhancer interactions. Cell 161, 1012-1025 (2015).

10. Ong, C. T. \& Corces, V. G. CTCF: an architectural protein bridging genome topology and function. Nat. Rev. Genet. 15, 234-246 (2014).

11. Phillips-Cremins, J. E. et al. Architectural protein subclasses shape 3D organization of genomes during lineage commitment. Cell 153, 1281-1295 (2013).

12. Rao, S. S. et al. A 3D map of the human genome at kilobase resolution reveals principles of chromatin looping. Cell 159, 1665-1680 (2014).

13. Sanborn, A. L. et al. Chromatin extrusion explains key features of loop and domain formation in wild-type and engineered genomes. Proc. Natl Acad. Sci. USA 112, E6456-E6465 (2015)

14. Vietri Rudan, M. et al. Comparative Hi-C reveals that CTCF underlies evolution of chromosomal domain architecture. Cell Rep. 10, 1297-1309 (2015).
15. Vietri Rudan, M. \& Hadjur, S. Genetic tailors: CTCF and cohesin shape the genome during evolution. Trends Genet. 31, 651-660 (2015).

16. Nora, E. P. et al. Targeted degradation of CTCF decouples local insulation of chromosome domains from genomic compartmentalization. Cell 169, 930-944 (2017). e22.

17. Franke, M. et al. Formation of new chromatin domains determines pathogenicity of genomic duplications. Nature 538, 265-269 (2016).

18. Rodriguez-Carballo, E. et al. The HoxD cluster is a dynamic and resilient TAD boundary controlling the segregation of antagonistic regulatory landscapes. Genes Dev. 31, 2264-2281 (2017).

19. Hacisuleyman, E. et al. Topological organization of multichromosomal regions by the long intergenic noncoding RNA Firre. Nat. Struct. Mol. Biol. 21, 198-206 (2014).

20. Hacisuleyman, E., Shukla, C. J., Weiner, C. L. \& Rinn, J. L. Function and evolution of local repeats in the Firre locus. Nat. Commun. 7, 11021 (2016).

21. Deng, X. et al. Bipartite structure of the inactive mouse X chromosome. Genome Biol. 16, 152 (2015).

22. Giorgetti, L. et al. Structural organization of the inactive $\mathrm{X}$ chromosome in the mouse. Nature 535, 575-579 (2016)

23. Darrow, E. M. et al. Deletion of DXZ4 on the human inactive X chromosome alters higher-order genome architecture. Proc. Natl Acad. Sci. USA 113 E4504-E4512 (2016).

24. da Rocha, S. T. \& Heard, E. Novel players in X inactivation: insights into Xistmediated gene silencing and chromosome conformation. Nat. Struct. Mol. Biol. 24, 197-204 (2017).

25. Jegu, T., Aeby, E. \& Lee, J. T. The X chromosome in space. Nat. Rev. Genet. 18, 377-389 (2017).

26. Bonora, G. et al. Orientation-dependent Dxz4 contacts shape the 3D structure of the inactive X chromosome. Preprint at https://www.biorxiv.org/content/ early/2017/12/15/165340 (2017).

27. Engreitz, J. M., Ollikainen, N. \& Guttman, M. Long non-coding RNAs: spatial amplifiers that control nuclear structure and gene expression. Nat. Rev. Mol. Cell. Biol. 17, 756-770 (2016).

28. Rinn, J. \& Guttman, M. RNA Function. RNA and dynamic nuclear organization. Science 345, 1240-1241 (2014).

29. Tan, J. Y. et al. cis-Acting complex-trait-associated lincRNA expression correlates with modulation of chromosomal architecture. Cell Rep. 18, 2280-2288 (2017)

30. Amaral, R. P. et al. Genomic positional conservation identifies topological anchor point RNAs linked to developmental loci. Genome Biol. 19, 32 (2018).

31. Kung, J. T. et al. Locus-specific targeting to the $\mathrm{X}$ chromosome revealed by the RNA interactome of CTCF. Mol. Cell. 57, 361-375 (2015).

32. Mele, M. \& Rinn, J. L. "Cat's Cradling" the 3D genome by the act of LncRNA transcription. Mol. Cell. 62, 657-664 (2016).

33. Andergassen, D. et al. Mapping the mouse Allelome reveals tissue-specific regulation of allelic expression. eLife 6, e25125 (2017).

34. Maass, P. G. et al. Spatiotemporal allele organization by allele-specific CRISPR live-cell imaging (SNP-CLING). Nat. Struct. Mol. Biol. 25, 176-184 (2018).

35. Naumova, N., Smith, E. M., Zhan, Y. \& Dekker, J. Analysis of long-range chromatin interactions using chromosome conformation capture. Methods $\mathbf{5 8}$, 192-203 (2012).

36. de Wit, E. et al. CTCF binding polarity determines chromatin looping. Mol. Cell. 60, 676-684 (2015).

37. Goloborodko, A., Imakaev, M. V., Marko, J. F. \& Mirny, L. Compaction and segregation of sister chromatids via active loop extrusion. eLife 5, e14864 (2016).

38. Rodriguez-Carballo, E. et al. The HoxD cluster is a dynamic and resilient TAD boundary controlling the segregation of antagonistic regulatory landscapes. Genes Dev. 31, 2264-2281 (2017).

39. Narendra, V. et al. CTCF establishes discrete functional chromatin domains at the Hox clusters during differentiation. Science 347, 1017-1021 (2015).

40. Rao, S. S. P. et al. Cohesin loss eliminates all loop domains. Cell 171, 305-320 (2017).

41. Schwarzer, W. et al. Two independent modes of chromatin organization revealed by cohesin removal. Nature 551, 51-56 (2017).

42. Beagan, J. A. et al. YY1 and CTCF orchestrate a 3D chromatin looping switch during early neural lineage commitment. Genome Res. 27, 1139-1152 (2017).

43. Weintraub, A. S. et al. YY1 is a structural regulator of enhancer-promoter loops. Cell 171, 1573-1588 (2017).

44. Rowley, M. J. et al. Evolutionarily conserved principles predict 3D chromatin organization. Mol. Cell. 67, 837-852 (2017).

45. Jeong, M. et al. A cell type-specific class of chromatin loops anchored at large DNA methylation nadirs. Preprint at https://www.biorxiv.org/content/early/ 2017/11/09/212928.1 (2017).

46. Barutcu, A. R. et al. SMARCA4 regulates gene expression and higher-order chromatin structure in proliferating mammary epithelial cells. Genome Res. 26, 1188-1201 (2016) 
47. Barutcu, A. R., Lian, J. B., Stein, J. L., Stein, G. S. \& Imbalzano, A. N. The connection between BRG1, CTCF and topoisomerases at TAD boundaries. Nucleus 8, 150-155 (2017).

48. Uuskula-Reimand, L. et al. Topoisomerase II beta interacts with cohesin and CTCF at topological domain borders. Genome Biol. 17, 182 (2016).

49. $\mathrm{Yu}, \mathrm{M}$. \& Ren, B. The three-dimensional organization of mammalian genomes. Annu. Rev. Cell. Dev. Biol. 33, 265-289 (2017).

50. Ou, H. D. et al. ChromEMT: Visualizing 3D chromatin structure and compaction in interphase and mitotic cells. Science 357, eaag0025 (2017)

51. Dixon, J. R., Gorkin, D. U. \& Ren, B. Chromatin domains: the unit of chromosome organization. Mol. Cell. 62, 668-680 (2016).

52. Durand, N. C. et al. Juicebox provides a visualization system for Hi-C contact maps with unlimited zoom. Cell Syst. 3, 99-101 (2016).

53. Durand, N. C. et al. Juicer provides a one-click system for analyzing loopresolution Hi-C experiments. Cell Syst. 3, 95-98 (2016).

54. Servant, N. et al. HiC-Pro: an optimized and flexible pipeline for $\mathrm{Hi}-\mathrm{C}$ data processing. Genome Biol. 16, 259 (2015).

55. Jung, Y. H. et al. Chromatin states in mouse sperm correlate with embryonic and adult regulatory landscapes. Cell Rep. 18, 1366-1382 (2017).

56. Wang, H. et al. Widespread plasticity in CTCF occupancy linked to DNA methylation. Genome Res. 22, 1680-1688 (2012).

57. Consortium, E. P. An integrated encyclopedia of DNA elements in the human genome. Nature 489, 57-74 (2012)

58. Niakan, K. K., Schrode, N., Cho, L. T. \& Hadjantonakis, A. K. Derivation of extraembryonic endoderm stem (XEN) cells from mouse embryos and embryonic stem cells. Nat. Protoc. 8, 1028-1041 (2013).

59. Langmead, B. \& Salzberg, S. L. Fast gapped-read alignment with Bowtie 2. Nat. Methods 9, 357-359 (2012)

60. Li, B. \& Dewey, C. N. RSEM: accurate transcript quantification from RNA-Seq data with or without a reference genome. BMC Bioinformatics 12, 323 (2011).

61. Love, M. I., Huber, W. \& Anders, S. Moderated estimation of fold change and dispersion for RNA-seq data with DESeq2. Genome Biol. 15, 550 (2014).

62. Lee, T. I., Johnstone, S. E. \& Young, R. A. Chromatin immunoprecipitation and microarray-based analysis of protein location. Nat. Protoc. 1, 729-748 (2006).

63. Heinz, S. et al. Simple combinations of lineage-determining transcription factors prime cis-regulatory elements required for macrophage and B cell identities. Mol. Cell. 38, 576-589 (2010).

64. Grant, C. E., Bailey, T. L. \& Noble, W. S. FIMO: scanning for occurrences of a given motif. Bioinformatics 27, 1017-1018 (2011).

65. Sandelin, A., Alkema, W., Engstrom, P., Wasserman, W. W. \& Lenhard, B. JASPAR: an open-access database for eukaryotic transcription factor binding profiles. Nucleic Acids Res. 32, D91-D94 (2004).

66. Belaghzal, H., Dekker, J. \& Gibcus, J. H. Hi-C. 2.0: An optimized Hi-C procedure for high-resolution genome-wide mapping of chromosome conformation. Methods 123, 56-65 (2017).

67. Keane, T. M. et al. Mouse genomic variation and its effect on phenotypes and gene regulation. Nature 477, 289-294 (2011).

68. Quinlan, A. R. \& Hall, I. M. BEDTools: a flexible suite of utilities for comparing genomic features. Bioinformatics 26, 841-842 (2010).

69. Crane, E. et al. Condensin-driven remodelling of X chromosome topology during dosage compensation. Nature 523, 240-244 (2015).

70. Doench, J. G. et al. Rational design of highly active sgRNAs for CRISPR-Cas9mediated gene inactivation. Nat. Biotechnol. 32, 1262-1267 (2014).
71. Shechner, D. M., Hacisuleyman, E., Younger, S. T. \& Rinn, J. L. Multiplexable, locus-specific targeting of long RNAs with CRISPR-Display. Nat. Methods 12, 664-670 (2015).

\section{Acknowledgements}

We thank the Rinn Lab members for useful discussions, especially Chiara Gerhardinger and James C. Lee for critical reading of the manuscript; Laurie Chen and Lin $\mathrm{Wu}$ at the Harvard Genome Modification Facility for their technical help with Firre KO mESC generation; and Christian Daly for the technical help with sequencing. Deep sequencing was performed at the Bauer Core Facility at Harvard University FAS Division of Science, the imaging was performed at $\mathrm{HCBI}$, and the computations in this study were performed on the Odyssey cluster supported by the FAS Division of Science, Research Computing Group at Harvard University. This study was funded by NIH grants U01 DA040612-01 and R01 MH102416. The Deutsche Forschungsgemeinschaft (DFG) supported P.G.M. (MA5028/1-3 and MA5028/1-1).

\section{Author contributions}

J.L.R. and A.R.B. devised the project. J.P.L. generated Firre KO transgenic mice and generated all the MEF lines. A.R.B. performed the Hi-C, 3C, ChIP-seq experiments, and performed all the bioinformatic analyses. P.G.M. and A.R.B. performed CRISPR live-cell imaging experiments. P.G.M. analyzed the imaging data. C.L.W. performed RNA-seq experiments. A.R.B. wrote the manuscript with input from J.P.L., P.G.M., and J.L.R.

\section{Additional information}

Supplementary Information accompanies this paper at https://doi.org/10.1038/s41467 018-03614-0.

Competing interests: The authors declare no competing interests.

Reprints and permission information is available online at http://npg.nature.com/ reprintsandpermissions/

Publisher's note: Springer Nature remains neutral with regard to jurisdictional claims in published maps and institutional affiliations.

Open Access This article is licensed under a Creative Commons Attribution 4.0 International License, which permits use, sharing, adaptation, distribution and reproduction in any medium or format, as long as you give appropriate credit to the original author(s) and the source, provide a link to the Creative Commons license, and indicate if changes were made. The images or other third party material in this article are included in the article's Creative Commons license, unless indicated otherwise in a credit line to the material. If material is not included in the article's Creative Commons license and your intended use is not permitted by statutory regulation or exceeds the permitted use, you will need to obtain permission directly from the copyright holder. To view a copy of this license, visit http://creativecommons.org/ licenses/by/4.0/.

(c) The Author(s) 2018 